Revista Brasil. Bot., V.31, n.4, p.621-632, out.-dez. 2008

\title{
Dinâmica do componente arbustivo-arbóreo de uma floresta de galeria aluvial no planalto de Poços de Caldas, MG, Brasil
}

\author{
JOÃO C. C. GUIMARÃES ${ }^{1,4}$, EDUARDO VAN DEN BERG², GISLENE C. CASTRO ${ }^{3}$, \\ EVANDRO L. M. MACHADO ${ }^{3}$ e ARY T. OLIVEIRA-FILHO ${ }^{3}$
}

(recebido: 17 de janeiro de 2008; aceito: 29 de agosto de 2008)

\begin{abstract}
Dynamics of the shrub-tree component of an alluvial gallery forest on the Poços de Caldas Plateau, MG State, Brazil). The dynamics of the shrub-tree component was investigated in an alluvial gallery forest in Poços de Caldas, Minas Gerais, from 1998 to 2005. The main objective of this study was to verify if the rates of the dynamics of the alluvial gallery forests are less than other tropical riparian forest formations. Seasonal flooding was also studied to see if it generates a spatial variation in the dynamics of going from the stream side towards the forest border. The sample consisted of 101, $10 \times 10$-meter plots that were distributed in five blocks extending approximately $8 \mathrm{~km}$ along the Antas River's forest. Each block was divided into three sectors (stream side, forest interior, and forest edge). Compared with other tropical forests, especially the riparian ones, this forest showed lower rates in terms of structural alterations. The forest edge is more dynamic, mainly due to the greater amount of sunlight, which supplies an adequate environment for species with a short life cycle.
\end{abstract}

Key words - anthropic impacts, seasonal flooding, self-thinning, water saturation

RESUMO - (Dinâmica do componente arbustivo-arbóreo de uma floresta de galeria aluvial no planalto de Poços de Caldas, MG, Brasil). A dinâmica do componente arbustivo-arbóreo foi investigada em uma floresta de galeria aluvial em Poços de Caldas, Minas Gerais, entre 1998 e 2005. Os objetivos principais foram: investigar se as taxas de dinâmica das florestas de galeria aluviais são inferiores a outras formações florestais ripárias tropicais e, se as inundações periódicas geram variação espacial na dinâmica no sentido Rio-Borda. A amostragem foi composta de 101 parcelas de $10 \times 10 \mathrm{~m}$, distribuídas em 5 blocos ao longo de, aproximadamente, $8 \mathrm{~km}$ da floresta do Rio das Antas. Cada bloco foi dividido em 3 setores (Rio, Interior e Borda). Comparativamente a outras florestas tropicais ripárias, a floresta em estudo apresentou taxas de dinâmica mais lentas. A Borda foi mais dinâmica que os outros setores, provavelmente devido à maior incidência luminosa, propiciando ambiente adequado às espécies de ciclo de vida curto.

Palavras-chave - autodesbaste, impactos antrópicos, inundação periódica, saturação hídrica

\section{Introdução}

Florestas aluviais são formações ripárias que sofrem inundações periódicas devido às variações dos cursos d'água e estão associadas às planícies que os acompanham (Rodrigues \& Shepherd 2000). As inundações saturam hidricamente os solos, causando restrições ao desenvolvimento de espécies arbóreas (Lobo \& Joly 2000, Barddal et al. 2004), e, portanto, espera-se que essas tipologias sofram menores mudanças estruturais ao longo do tempo relativamente a outras florestas que não têm esse evento. Espera-se, também, que esta formação sofra mudanças estruturais diferenciadas no

1. Companhia Geral de Minas, Alcoa Alumínio S.A., Caixa Postal 128, 37719-900 Poços de Caldas, MG, Brasil.

2. Universidade Federal de Lavras, Departamento de Biologia, 37200-000 Lavras, MG, Brasil.

3. Universidade de Brasília, Departamento de Engenharia Florestal, Caixa Postal 04357, 70919-970 Brasília, DF, Brasil.

4._Autor para correspondência: joao.guimaraes@alcoa.com.br sentido Borda-Rio, visto que a intensidade luminosa difere entre os setores (van den Berg \& Santos 2003), e que os efeitos de sedimentação e estresse hídrico ocasionado pelas cheias sazonais também variem ao longo da floresta, refletindo variação espacial da dinâmica florestal.

No Brasil, foram realizados diversos estudos visando detectar as mudanças estruturais ocorridas em florestas, bem como tentar compreender os fatores que causam tais alterações (Rankin-de-Merona et al. 1990, Felfili 1995a, b, Pagano et al. 1995, Oliveira-Filho et al. 1997, Laurance et al. 1998, Nascimento et al. 1999, van den Berg 2001, Guilherme et al. 2004, Damasceno-Junior et al. 2004, Werneck \& Franceschinelli 2004, Appolinário et al. 2005, Pinto \& Hay 2005, Oliveira-Filho et al. 2007), porém nenhum destes investigou as mudanças em florestas aluviais.

Especificamente, o presente estudo pretendeu esclarecer se a dinâmica em florestas aluviais possui padrão diferenciado, com taxas inferiores, em relação a outras tipologias florestais ripárias tropicais e se a 
dinâmica da floresta varia no sentido da margem do rio em direção à borda da floresta.

\section{Material e métodos}

Área de estudo - O Rio das Antas localiza-se na porção sul do planalto de Poços de Caldas, Minas Gerais, entre as coordenadas $21^{\circ} 51^{\prime} 08^{\prime \prime}-21^{\circ} 55^{\prime} 48^{\prime \prime}$ de latitude sul e $46^{\circ} 34^{\prime} 53^{\prime \prime}-46^{\circ} 35^{\prime} 46^{\prime \prime}$ de longitude oeste, com altitude variando entre $1.253-1.265 \mathrm{~m}$.

O clima é do tipo Cwb, subtropical de altitude, segundo a classificação de Köppen, mesotérmico com verões brandos e estiagem de inverno. O índice pluviométrico anual em Poços de Caldas é de $1.482 \mathrm{~mm}$, sendo que a estação chuvosa estende-se de outubro a março. A temperatura média anual é de $19,9^{\circ} \mathrm{C}$, sendo que as temperaturas mínimas e máximas absolutas são respectivamente $-6^{\circ} \mathrm{C}$ e $31,7^{\circ} \mathrm{C}$. Umidade relativa média anual de 79\% (Prefeitura Municipal de Poços de Caldas 1992).

O embasamento rochoso do planalto de Poços de Caldas é formado por rochas nefelíticas, tinguaitos e foiaitos, apresentando ainda rochas vulcânicas constituídas por tufos, brechas e conglomerados (Radambrasil 1983). A definição de Jacomine (2000) sobre os solos aluviais, ou neossolos flúvicos (Embrapa 2000), caracteriza bem o solo da floresta às margens do Rio das Antas. Segundo este autor, os solos aluviais ocorrem associados a florestas de galeria menos úmidas. Assim, tais solos ocorrem em áreas de terraços ou várzeas mais enxutas, sujeitas ao encharcamento por eventuais inundações. Tais solos são pouco desenvolvidos, apresentando apenas um horizonte "A" sobre camadas estratificadas sem relação pedogenética entre si. São profundos, com drenagem moderada e imperfeita, e com textura muito variável, devido à natureza dos sedimentos fluviais depositados.

A vegetação que ocorre na área de estudos pode ser definida como floresta de galeria. Florestas de galeria constituem estreitas faixas de floresta tropical ao longo dos córregos (Felfili 1995a, b) e rios de pequeno porte, formando corredores (galerias) sobre o curso d'água (Ribeiro \& Walter 1998). A floresta apresenta limites abruptos e bem definidos com formações savânicas e campestres em ambas as margens (Ribeiro \& Walter 1998). A floresta de galeria presente na área de estudos possui elevada deciduidade em vários trechos, chegando a ser superior a 50\% na estação seca (Jacomine 2000 e dados não publicados).

Apesar da topografia relativamente plana da floresta do Rio das Antas, ocorre a formação de uma micro-elevação (dique sedimentar) nas áreas marginais ao curso d'água devido ao maior acúmulo de sedimentos nesses locais durante as cheias.

Foram inventariados cinco blocos amostrais distribuídos ao longo de $8 \mathrm{~km}$ da floresta aluvial do Rio das Antas, denominados, em direção a nascente do rio, como AL, MG, EJ, RJ e DR (figura 1). Nos blocos AL e MG, foram encontradas cepas de árvores provenientes de corte seletivo e anteriores

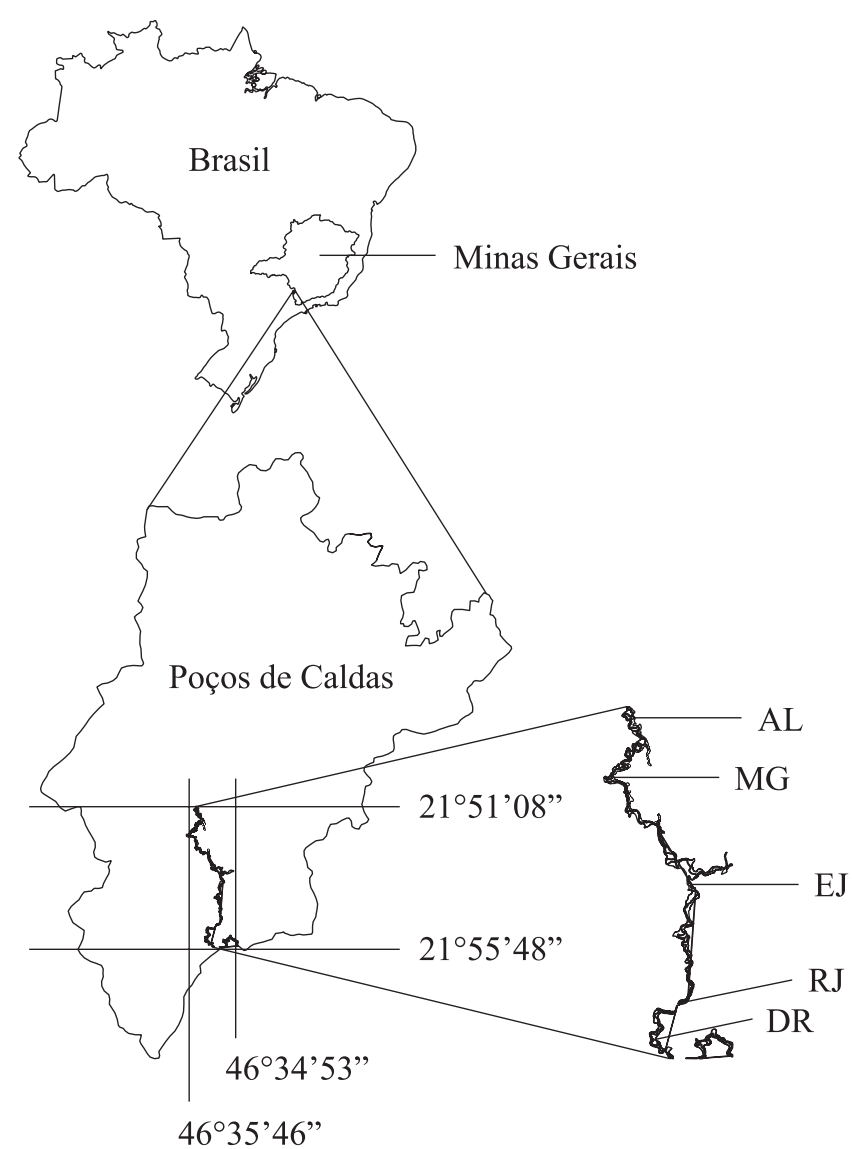

Figura 1. Localização dos blocos AL, MG, EJ, RJ e DR ao longo da floresta de galeria aluvial do Rio das Antas, Poços de Caldas, MG, Brasil.

Figure 1. Location of blocks AL, MG, EJ, RJ and DR in the Antas River gallery forest, Poços de Caldas, Minas Gerais State, Brazil.

ao primeiro inventário. Outro impacto visível, que atinge principalmente o bloco MG, é o tráfego e pastoreio de gado no sub-bosque. Um distúrbio de origem natural que atinge todos os blocos são as inundações periódicas e rápidas, as quais ocorrem na estação chuvosa, sendo que em alguns trechos ao longo do Rio das Antas ocorrem lagoas temporárias ou permanentes fora dos limites da floresta de galeria.

Inventários do componente arbustivo-arbóreo - Em 1998, a partir de ortofotos de 1997 e visitas à área, foram escolhidos cinco trechos da floresta de galeria ao longo do Rio das Antas. Em cada um desses trechos foi alocada uma transecção com $40 \mathrm{~m}$ de largura, cortando perpendicularmente a floresta, da borda desta com a vegetação circundante até o curso d'água. Cada transecção foi dividida em parcelas de $10 \times 10 \mathrm{~m}$ e denominada de bloco. Em cada bloco, a floresta foi subdivida em três setores ou hábitats (figura 2): Borda, composto pelas parcelas limítrofes com a vegetação não florestal circundante; Interior, composto pelas parcelas 
localizadas na porção intermediária da floresta; e Rio, definido pelas parcelas locadas junto ao curso d'água. O critério utilizado na definição destes três setores foi a diferença nas características ambientais, sendo que as parcelas localizadas mais próximas ao limite da floresta com o campo sofrem maior intensidade do "efeito borda", ou seja, maior intensidade luminosa, menor umidade relativa do ar, maior variação da temperatura, além da maior exposição aos efeitos dos ventos (van den Berg \& Santos 2003). Além disso, as parcelas próximas ao rio estão localizadas em áreas que apresentam maior deposição de sedimentos sendo, assim, ligeiramente mais elevadas que as demais, resultando em um lençol freático provavelmente mais profundo (E. van den Berg dados não publicados).

Todos os indivíduos de espécies arbóreas e arbustivas tiveram medidas com fita métrica suas circunferências a altura do solo (CAS) quando igual ou superior a $15,7 \mathrm{~cm}$, assim como suas alturas estimadas. Esses indivíduos foram marcados com plaquetas de alumínio numeradas e deles coletado material botânico para posterior identificação. A
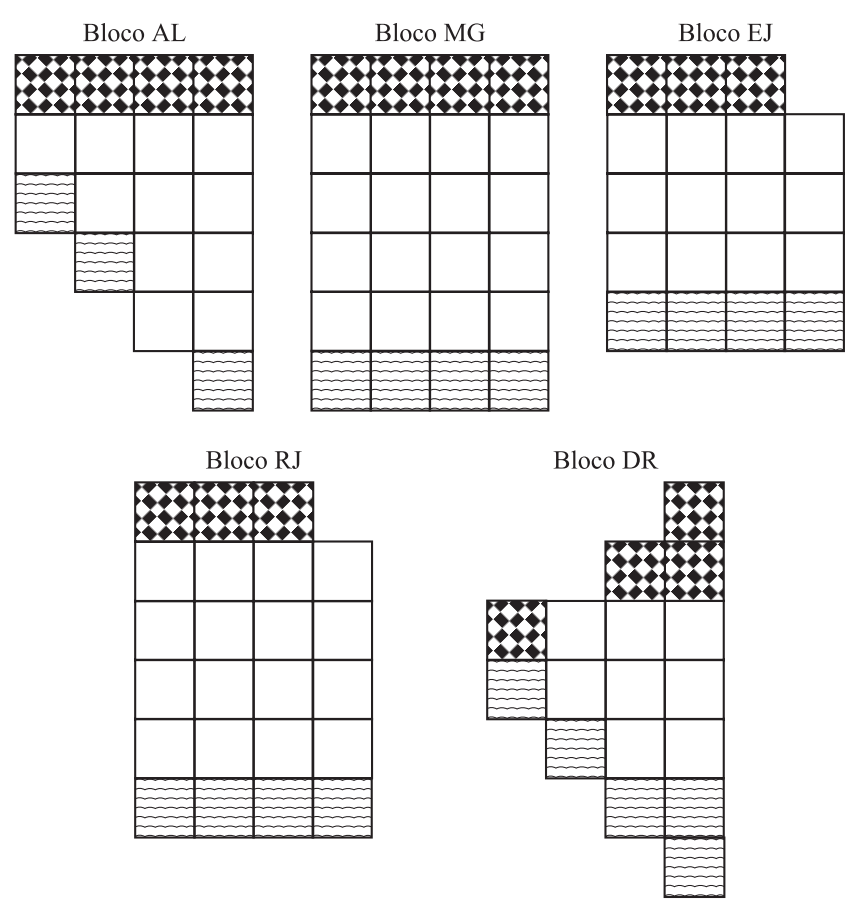

Figura 2. Distribuição das parcelas dos cinco blocos onde foram inventariados os indivíduos do componente arbustivo-arbóreo da floresta de galeria aluvial do Rio das Antas, Poços de Caldas, MG, Brasil, entre 1998 e 2005. (誹= Parcelas do Setor Borda; $\square=$ Parcelas do Setor Interior; 冒 = Parcelas do Setor Rio)

Figure 2. Distribution of the plots in the five blocks of the shrub-tree component that were sampled between 1988 and 2005, in the Antas River gallery forest, Poços de Caldas, Minas Gerais State, Brazil. ( = Forest Edge Sector's Plots; $\square=$ Forest Interior Sector's Plots; 后 = Stream Side Sector's Plots). identificação foi realizada por meio de observações de campo, com o auxílio da literatura e de especialistas e, quando necessário, por comparações com material do Herbário ESAL pertencente à UFLA. Uma exsicata de cada espécie foi inserida no acervo do Herbário.

Em 2005, 6,5 anos após o primeiro inventário, foi realizado um novo inventário utilizando os critérios supracitados. Os indivíduos que atingiram a dimensão de inclusão (recrutas) foram mensurados, assim como os sobreviventes. Os indivíduos mortos foram registrados.

Dinâmica florestal - Taxas de dinâmica foram calculadas para a amostra total, blocos e setores. As taxas expressam a dinâmica de duas formas: em número de indivíduos e em área basal, esta calculada pela soma das áreas seccionais a altura do solo, assumindo uma relação circular dos caules. Baseado em Sheil et al. (1995, 2000), foram assumidas mudanças no tamanho populacional por intervalo de tempo em proporção constante do tamanho, sendo que para a mortalidade foi tomado como referência o tamanho inicial da população, e para o recrutamento a base foi o tamanho final. Assim foram calculadas as taxas anuais médias de mortalidade $(\mathrm{M})$ e recrutamento $(\mathrm{R})$ de árvores individuais e taxas anuais médias de perda $(\mathrm{Pe})$ e ganho $(\mathrm{G})$ de área basal das árvores por meio das expressões exponenciais:

$$
\begin{aligned}
& M=\left\{1-\left[\left(N_{0}-N_{m}\right) / N_{o}\right]^{1 / t}\right\} \times 100 \\
& R=\left[1-\left(1-N_{r} / N_{t}\right)^{1 / t}\right] \times 100 \\
& P e=\left\{1-\left[\left(A B_{0}-A B_{m}-A B_{d}\right) / A B_{0}\right]^{1 / t}\right\} \times 100 \\
& G=\left\{1-\left[1-\left(A B_{r}+A B_{g}\right) / A B_{t}\right]^{1 / t}\right\} \times 100
\end{aligned}
$$

Onde: t é o tempo decorrido em anos entre os inventários; $\mathrm{N}_{0}$ e $\mathrm{N}_{\mathrm{t}}$ são, respectivamente, as contagens inicial e final de árvores individuais; $\mathrm{N}_{\mathrm{m}}$ e $\mathrm{N}_{\mathrm{r}}$ são, respectivamente, o número de árvores mortas e recrutas; $\mathrm{AB}_{0}$ e $\mathrm{AB}_{\mathrm{t}}$ são, respectivamente, as áreas basais inicial e final das árvores; $\mathrm{AB}_{\mathrm{m}}$ é a área basal das árvores mortas; $\mathrm{AB}_{\mathrm{r}}$ é a área basal dos recrutas; e $\mathrm{AB}_{\mathrm{d}} \mathrm{e}$ $\mathrm{AB}_{\mathrm{g}}$ são, respectivamente, o decremento (por meio de quebra ou perda parcial do tronco) e o incremento em área basal das árvores sobreviventes. Para expressar a dinâmica global, foram calculadas a partir das médias das taxas de mortalidade e recrutamento e de perda e ganho, respectivamente, as taxas de rotatividade ("turnover") em número de árvores $\left(\mathrm{T}_{\mathrm{N}}\right)$ e em área basal $\left(\mathrm{T}_{\mathrm{AB}}\right)$ (Oliveira-Filho et al. 1997, Werneck \& Franceschinelli 2004):

$$
\begin{aligned}
& T_{N}=(M+R) 2^{-1} \\
& T_{A B}=(P e+G) 2^{-1}
\end{aligned}
$$

Conforme Korning \& Balslev (1994), foram obtidas as taxas de mudança líquida no período, tanto para número de árvores $\left(\mathrm{Ch}_{\mathrm{N}}\right)$ como área basal $\left(\mathrm{Ch}_{\mathrm{AB}}\right)$, sendo utilizadas as equações:

$$
\begin{aligned}
& C h_{N}=\left[\left(N_{t} / N_{0}\right)^{1 / t}-1\right] \times 100 \\
& C h_{A B}=\left[\left(A B_{t} / A B_{0}\right)^{1 / t}-1\right] \times 100
\end{aligned}
$$


Após o calculo das taxas de dinâmica para cada parcela, foram realizadas comparações pela análise de variância não paramétrica de Kruskal-Wallis (Sokal \& Rohlf 1995) entre os blocos e entre os setores.

Após a transformação dos dados através do arco seno, foram testadas, por meio de regressão linear, relações entre as taxas de dinâmica em relação ao número inicial de indivíduos e à área basal inicial. Esse procedimento foi realizado para a amostra total, blocos e setores. A regressão permite inferir se a densidade e, ou, a área basal constatada no primeiro inventário, afetou a dinâmica da floresta em relação ao recrutamento e mortalidade de indivíduos, e ao ganho e perda em biomassa, no intervalo compreendido pelo estudo (Sokal \& Rohlf 1995).

Dinâmica nas diferentes classes diamétricas - A dinâmica das árvores por classe diamétrica na amostra total, nos cinco blocos e nos três setores, foi analisada empregando-se os mesmos intervalos de classe com amplitudes crescentes que foram adotados em estudos de dinâmica florestal na região do Alto Rio Grande (Appolinário et al. 2005, Oliveira-Fiho et al. 2007), para compensar o acentuado decréscimo da densidade nas classes de tamanhos maiores, típico da distribuição em exponencial negativo ( $J$-invertido). Para descrever as variações temporais ocorridas em cada classe de diâmetro, foi contabilizado o número de árvores que passou pelos seguintes eventos: permanência na classe, morte, recrutamento, imigração (ingrowth) e emigração (outgrowth) na classe, podendo os dois últimos ser progressivos ou regressivos (Lieberman et al. 1985). Com base na freqüência de indivíduos do primeiro inventário foram calculadas as freqüências esperadas para as classes diamétricas, conforme a fórmula descrita abaixo:

$$
N_{\text {esp }} \text { classe }=\left(N_{0} \text { classe } / N_{0} \text { total }\right) \times N_{t} \text { total }
$$

Onde: $\mathrm{N}_{\text {esp }}$ classe é o número de indivíduos esperado por classe diamétrica; $\mathrm{N}_{0}$ classe e $\mathrm{N}_{0}$ total são, respectivamente, as contagens iniciais de árvores por classe diamétrica e para o total do estrato; e $\mathrm{N}_{\mathrm{t}}$ total é a contagem final total de árvores para o estrato em questão.

Após essa etapa foi verificado, usando o teste de quiquadrado $\left(\chi^{2}\right)$, se as freqüências observadas no segundo inventário foram diferentes dos valores esperados. Com base na distribuição diamétrica em 1998, foram utilizados testes de qui-quadrado $\left(\chi^{2}\right)$ para verificar se as freqüências de árvores vivas em 2005 e de árvores mortas no período de 6,5 anos foram independentes das classes de diâmetro. A diferença entre o número de árvores ingressantes (recrutas + imigrantes) e árvores egressas (mortos + emigrantes) em cada classe de diâmetro foi verificada por comparações entre contagens de Poisson (Zar 1996).

\section{Resultados}

Dinâmica em termos de número de indivíduos e área basal - Os resultados de dinâmica encontram-se na tabela 1 .
Para a amostra total, a taxa de mudança líquida em número de indivíduos foi de $-0,61 \%$ ano $^{-1}$. Esta taxa foi significativamente diferente entre os blocos $(P=0,0015)$, sendo que o bloco AL foi o que apresentou a maior perda de indivíduos $\left(-1,18 \% \mathrm{ano}^{-1}\right)$. O bloco RJ apresentou maior estabilidade $\left(0,02 \% \mathrm{ano}^{-1}\right)$, sendo que, entre todos os blocos, este foi o único que apresentou taxa positiva. A taxa de recrutamento também apresentou diferença significativa entre os blocos $(P=0,0001)$, sendo que o menor valor foi detectado no bloco $\operatorname{AL}\left(0,67 \% \mathrm{ano}^{-1}\right) \mathrm{e}$ o maior no bloco DR (2,19\% ano $\left.{ }^{-1}\right)$. A taxa de rotatividade também foi estatisticamente diferente entre os blocos ( $P=0,0041)$, sendo que o bloco AL apresentou o menor valor $\left(1,26 \%\right.$ ano $\left.^{-1}\right)$ e o DR apresentou o maior valor $\left(2,33 \% \mathrm{ano}^{-1}\right)$. A comparação das taxas de mudança líquida em número de indivíduos dos setores indicou que há diferença significativa $(P=0,0138)$, sendo possível indicar a ocorrência de um gradiente com um aumento progressivo da perda de indivíduos do setor Rio (-0,04\% ano $\left.^{-1}\right)$ até o setor Borda $\left(-1,14 \%\right.$ ano $\left.^{-1}\right)$. A diferença significativa na taxa de mudança líquida em densidade entre os setores refletiu em diferenças significativas para as demais taxas de dinâmica para densidade (M: $P=0,0026$; R: $\left.P=0,0273 ; \mathrm{T}_{\mathrm{N}}: P=0,0008\right)$. O setor Rio apresentou a menor taxa de mortalidade $\left(1,48 \%\right.$ ano $\left.^{-1}\right)$, enquanto o setor Interior apresentou as menores taxas de recrutamento $\left(1,12 \%\right.$ ano $\left.^{-1}\right)$ e rotatividade $\left(1,43 \%\right.$ ano $\left.^{-1}\right)$. O setor Borda apresentou as parcelas com as maiores taxas de dinâmica $\left(\mathrm{M}=2,75 \%\right.$ ano $\left.^{-1} ; \mathrm{R}=1,63 \% \mathrm{ano}^{-1} ; \mathrm{T}_{\mathrm{N}}=2,19 \% \mathrm{ano}^{-1}\right)$.

A área basal da amostra total apresentou crescimento no intervalo entre os dois levantamentos $\left(\mathrm{Ch}_{\mathrm{AB}}=1,67 \%\right.$ ano $\left.^{-1}\right)$. Houve diferenças significativas para taxa de mudança líquida entre os blocos $(P=0,0022)$, sendo que o bloco $\mathrm{MG}$ apresentou menor valor para esta taxa $\left(1,01 \%\right.$ ano $\left.^{-1}\right)$, enquanto o oposto ocorreu com o bloco DR (2,28\% ano $\left.{ }^{-1}\right)$. Os setores apresentaram diferença significativa para todas as taxas de dinâmica relacionadas com a área basal, exceto para a taxa de mudança (Pe: $\left.P=0,0334 ; \mathrm{G}: P=0,0041 ; \mathrm{T}_{\mathrm{AB}}: P=0,0011\right)$. Os setores Interior e Rio apresentaram taxas de dinâmica em área basal semelhantes, sendo que o setor Borda apresentou taxas de dinâmica mais aceleradas que os demais $(\mathrm{Pe}=$ $1,62 \%$ ano $^{-1} ; \mathrm{G}=3,53 \% \mathrm{ano}^{-1} ; \mathrm{T}_{\mathrm{AB}}=2,58 \% \mathrm{ano}^{-1} ; \mathrm{Ch}_{\mathrm{AB}}=$ $\left.1,97 \% \mathrm{ano}^{-1}\right)$.

Taxas de dinâmica versus densidade e área basal inicial Os resultados estatisticamente significativos encontram-se na tabela 2. Para a amostra total foi encontrada relação negativa significativa entre o número inicial de indivíduos e a taxa de mudança líquida em densidade $(b=-0,147$; $P=0,0247)$. O mesmo ocorreu no setor Borda $(b=-0,270$; 


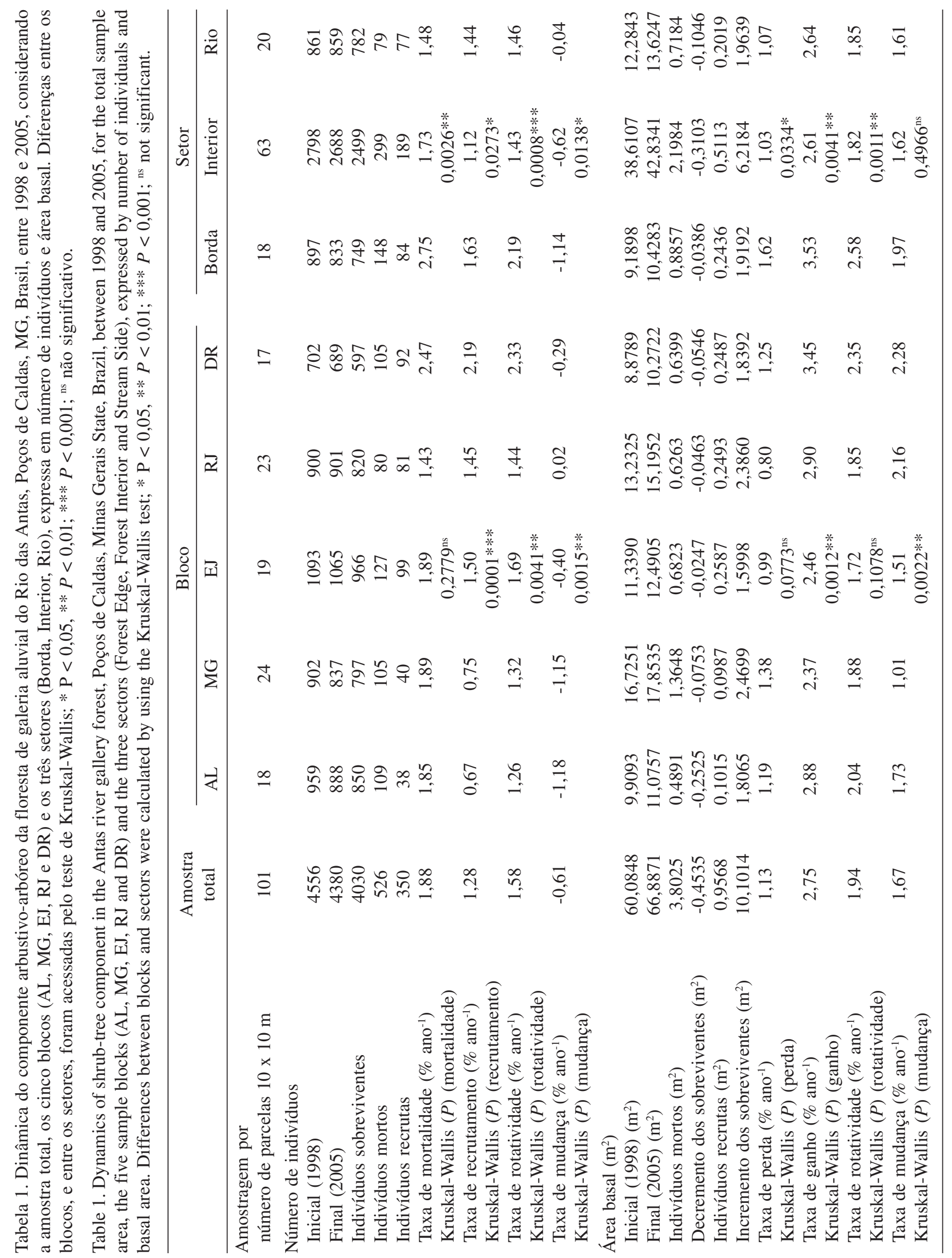


$P=0,0334)$, e nos blocos EJ $(\mathrm{b}=-0,223 ; P=0,0243)$ e $\mathrm{DR}(\mathrm{b}=-0,651 ; P=0,0051)$. A densidade inicial também se relacionou negativamente com a taxa de mudança líquida em área basal no setor Borda $(\mathrm{b}=-0,155 ; P=$ $0,0151)$ e no bloco DR $(b=-0,501 ; P=0,0002)$. A regressão entre o número inicial de indivíduos e a taxa de mudança líquida em área basal para o bloco DR foi a que teve o maior coeficiente de determinação $\left(R^{2}=0,6144\right)$ e a maior relação estatística pelo teste F. Apenas nas parcelas do bloco AL, a área basal inicial afetou negativamente a taxa de mudança líquida em área basal de forma significativa $(b=-19,340 ; P=0,0317)$.

A taxa de recrutamento foi afetada significativamente e de forma negativa pelo número inicial de indivíduos no setor Borda $(\mathrm{b}=-0,081 ; P=0,0387)$ e no bloco RJ $(\mathrm{b}=-0,242 ; P=0,0297)$, e pela área basal inicial no setor Borda $(\mathrm{b}=-12,030 ; P=0,0102)$ e na amostra total $(\mathrm{b}=-5,435 ; P=0,0498)$, sendo que para esta regressão

Tabela 2. Regressões lineares estatisticamente significativas para amostra total, blocos e setores do componente arbustivoarbóreo da floresta de galeria aluvial do Rio das Antas, Poços de Caldas, MG, Brasil, entre 1998 e 2005, relacionando o número de indivíduos inicial e a área basal inicial com as taxas de dinâmica $(\mathrm{Ni}=$ número inicial de indivíduos; $\mathrm{ABi}=$ área basal inicial; $\mathrm{x}=$ variável independente; $\mathrm{y}=\mathrm{a}$ variável dependente; Equação $=$ equação de regressão linear; $\mathrm{R}^{2}=$ coeficiente de determinação; $\mathrm{F}=\mathrm{o}$ valor do teste $\mathrm{F} ; P=$ nível de significância); $* P<0,05 ; * * P<0,01$; *** $P<0,001$.

Table 2. Statistically significant linear regressions for the total sample, blocks and sectors for the shrub-tree component of Antas river gallery forest, Poços de Caldas, Minas Gerais State, Brazil, between 1998 and 2005, relating the initial number of individuals and the basal area to the dynamic rates $(\mathrm{Ni}=$ initial number of individuals; $\mathrm{ABi}=$ initial basal area; $\mathrm{x}=$ independent variable; $\mathrm{y}=$ dependent variable; Equation $=$ linear regression equation; $\mathrm{R}^{2}=$ determination coefficient; $\mathrm{F}=\mathrm{F}$ test value; $P=$ significance level); $* P<0,05 ; * * P<0,01 ; * * * P<0,001$.

\begin{tabular}{|c|c|c|c|c|c|}
\hline $\mathrm{x}$ & $\mathrm{y}$ & Equação & $\mathrm{R}^{2}$ & $\mathrm{~F}$ & $P$ \\
\hline \multicolumn{6}{|c|}{ Amostra total } \\
\hline $\mathrm{Ni}$ & Taxa de perda & $y=6,639+0,073^{*} x$ & 0,0728 & 7,77 & $0,0064 * *$ \\
\hline $\mathrm{Ni}$ & Taxa de mudança $\mathrm{N}$ & $y=2,273-0,147^{*} x$ & 0,0499 & 5,19 & $0,0247 *$ \\
\hline $\mathrm{ABi}$ & Taxa de recrutamento & $y=13,399-5,435^{*} x$ & 0,0383 & 3,95 & $0,0498^{*}$ \\
\hline $\mathrm{ABi}$ & Taxa de ganho & $y=19,894-5,468 * x$ & 0,1186 & 13,32 & $0,0004 * * *$ \\
\hline \multicolumn{6}{|c|}{ Bloco AL } \\
\hline $\mathrm{ABi}$ & Taxa de mortalidade & $y=25,179-21,750 * x$ & 0,2359 & 4,94 & $0,0409 *$ \\
\hline $\mathrm{ABi}$ & Taxa de ganho & $y=23,860-12,260 * x$ & 0,2980 & 6,79 & $0,0191 *$ \\
\hline $\mathrm{ABi}$ & Taxa de mudança AB & $y=23,506-19,360 * x$ & 0,2573 & 5,54 & $0,0317 *$ \\
\hline \multicolumn{6}{|c|}{ Bloco EJ } \\
\hline $\mathrm{Ni}$ & Taxa de perda & $y=4,254+0,087 * x$ & 0,3273 & 8,27 & $0,0105^{*}$ \\
\hline $\mathrm{Ni}$ & Taxa de mudança $\mathrm{N}$ & $y=10,711-0,223^{*} x$ & 0,2644 & 6,11 & $0,0243^{*}$ \\
\hline \multicolumn{6}{|c|}{ Bloco RJ } \\
\hline $\mathrm{Ni}$ & Taxa de recrutamento & $y=20,852-0,242 * x$ & 0,2057 & 5,44 & $0,0297 *$ \\
\hline \multicolumn{6}{|c|}{ Bloco DR } \\
\hline $\mathrm{Ni}$ & Taxa de mortalidade & $y=2,675+0,282 * x$ & 0,4734 & 13,48 & $0,0023 * *$ \\
\hline $\mathrm{Ni}$ & Taxa de perda & $y=-2,203+0,307 * x$ & 0,5642 & 19,42 & $0,0005^{* *}$ \\
\hline $\mathrm{Ni}$ & Taxa de mudança $\mathrm{N}$ & $y=26,340-0,651 * x$ & 0,4171 & 10,73 & $0,0051 * *$ \\
\hline $\mathrm{Ni}$ & Taxa de mudança $\mathrm{AB}$ & $y=34,770-0,501 * x$ & 0,6144 & 23,90 & $0,0002 * * *$ \\
\hline $\mathrm{ABi}$ & Taxa de ganho & $y=25,693-13,200 * x$ & 0,3120 & 6,80 & $0,0198^{*}$ \\
\hline \multicolumn{6}{|c|}{ Setor Borda } \\
\hline $\mathrm{Ni}$ & Taxa de recrutamento & $\mathrm{y}=17,050-0,081 * x$ & 0,2408 & 5,08 & $0,0387^{*}$ \\
\hline $\mathrm{Ni}$ & Taxa de mudança $\mathrm{N}$ & $y=8,512-0,270 * x$ & 0,2528 & 5,41 & $0,0334 *$ \\
\hline $\mathrm{Ni}$ & Taxa de mudança $\mathrm{AB}$ & $y=21,142-0,155 * x$ & 0,3165 & 7,41 & $0,0151 *$ \\
\hline $\mathrm{ABi}$ & Taxa de recrutamento & $y=19,140-12,030 * x$ & 0,3462 & 8,47 & $0,0102 *$ \\
\hline \multicolumn{6}{|c|}{ Setor Interior } \\
\hline $\mathrm{ABi}$ & Taxa de ganho & $y=22,932-7,216 * x$ & 0,1919 & 14,48 & $0,0003 * * *$ \\
\hline
\end{tabular}


foi obtido o menor coeficiente de determinação $\left(R^{2}=\right.$ 0,0383 ) entre todas as regressões testadas que foram significativas estatisticamente. A taxa de mortalidade foi afetada negativamente pela área basal inicial no bloco AL ( $\mathrm{b}=-21,750 ; P=0,0410)$, e positivamente pelo número inicial de indivíduos no bloco $\mathrm{DR}(\mathrm{b}=0,282$; $P=0,0023)$.

A taxa de perda em área basal foi afetada positivamente, em termos estatísticos, de forma significativa pelo número inicial de indivíduos nas parcelas da amostra total $(\mathrm{b}=$ $0,073 ; P=0,0064)$, e dos blocos EJ $(b=0,087 ; P=0,0105)$ e DR ( $b=0,307 ; P=0,0005)$. A taxa de ganho foi afetada negativamente pela área basal inicial na amostra total $(\mathrm{b}=-5,468 ; P=0,0004)$, no setor Interior $(\mathrm{b}=-7,216$; $P=0,0003)$, e nos blocos AL ( $b=-12,260 ; P=0,0191)$ e DR ( $b=-13,200 ; P=0,0198)$. Somente o setor Rio e o bloco MG não tiveram nenhuma relação significativa entre as taxas de dinâmica e o número inicial de indivíduos e, ou a área basal inicial.

Dinâmica nas diferentes classes diamétricas - A distribuição das freqüências de indivíduos observadas por classes diamétricas em 2005 diferiu significativamente da distribuição esperada em relação a 1998 para a amostra total, para os blocos AL, EJ e DR, e para os setores Interior e Borda. Quando a comparação foi realizada entre as frequiências observada e esperada para a contagem de indivíduos mortos por classe diamétrica no intervalo entre os dois inventários, a significância foi ainda maior, sendo que neste caso somente o bloco $\mathrm{MG}$ não apresentou diferença significativa (tabela 3 ).

A análise da primeira classe diamétrica $(5 \leq$ DAS $<10 \mathrm{~cm}$ ) em separado indicou que o número de indivíduos egressos (mortos mais emigrantes) superou o de ingressantes (recrutas mais imigrantes) na amostra total e em todos os blocos e setores, excetuando-se o bloco RJ e o setor Rio. Para a segunda classe $(10 \leq$ DAS $<20 \mathrm{~cm}$ ), no bloco MG o número de indivíduos egressos superou o de ingressantes $(\mathrm{Z}=2,86 ; P=0,005)$, e no bloco AL ( $\mathrm{Z}=2,98 ; P=0,005)$ e no setor Borda ( $\mathrm{Z}=2,59 ; P=0,01)$ ocorreu um ganho líquido de indivíduos. Na terceira classe $(20 \leq$ DAS $<40 \mathrm{~cm})$ todas as contagens de Poisson foram significativas e em todas as situações ocorreu ganho líquido de indivíduos (tabela 3). Nas demais classes não ocorrem contagens significativas.

Tabela 3. Dinâmica por classe diamétrica do componente arbustivo-arbóreo da floresta de galeria aluvial do Rio das Antas, Poços de Caldas, MG, Brasil, entre 1998 e 2005, contabilizada para a amostra total, blocos e setores. As freqüências esperadas para o número total de indivíduos e para o número de mortos por classe de Diâmetro a Altura do Solo (DAS) em 2005 basearam-se na distribuição do número de indivíduos por classe de DAS em 1998 (esp. = freqüência esperada em número de indivíduos; Emig. = emigrantes; Rec. = recrutas; Imig. = imigrantes; Cont. Poisson = contagens de Poisson para saídas de indivíduos, mortos + emigrantes, e entradas de indivíduos, recrutas + imigrantes); ${ }^{*} P<0,05 ;{ }^{*} * P<0,01 ;{ }^{\text {ns }}$ não significativo.

Table 3. Dynamics by class of diameters of the shrub-tree component of the Antas river gallery forest, Poços de Caldas, Minas Gerais State, Brazil, between 1998 and 2005, for the total sample, blocks and sectors. The expected frequencies for the total number of individuals at and for the number of dead individuals by Diameters at Soil Level (DAS) class in 2005 were based on the distribution of individuals by DAS class in 1998 (esp. = expected frequencies for the number of individuals; Emig. = emigrants; Rec. = recruits; Imig. = immigrants; Cont. Poisson $=$ Poisson counting for outgrowth, dead individuals + emigrants, and ingrowth, recruits + immigrants); $*<<0,05 ; * * P<0,01$; ${ }^{\text {ns }}$ not significant.

\begin{tabular}{|c|c|c|c|c|c|c|c|c|c|c|c|}
\hline \multirow{2}{*}{$\begin{array}{l}\text { DAS } \\
(\mathrm{cm})\end{array}$} & \multicolumn{3}{|c|}{ № de árvores } & \multicolumn{3}{|c|}{ Mortos } & \multirow{2}{*}{$\frac{\text { Emig. }}{\text { № }}$} & \multirow{2}{*}{$\frac{\text { Rec. }}{\text { № }}$} & \multirow{2}{*}{$\frac{\text { Imig. }}{\text { № }}$} & \multicolumn{2}{|c|}{ Cont. Poisson } \\
\hline & 1998 & 2005 & esp. & № & esp. & $\% \mathrm{ano}^{-1}$ & & & & $\mathrm{Z}$ & $P$ \\
\hline \multicolumn{12}{|c|}{ Amostra total } \\
\hline $5<10$ & 2651 & 2360 & 2549 & 420 & 306 & 2,63 & 238 & 350 & 17 & 9,09 & 0,001 \\
\hline $10<20$ & 1510 & 1528 & 1452 & 92 & 174 & 0,97 & 133 & 0 & 243 & 0,83 & ns \\
\hline $20<40$ & 363 & 454 & 349 & 12 & 42 & 0,52 & 13 & 0 & 116 & 7,66 & 0,001 \\
\hline $40<80$ & 31 & 37 & 30 & 2 & 4 & 1,03 & 0 & 0 & 8 & 1,90 & ns \\
\hline$>80$ & 1 & 1 & 1 & 0 & 0 & 0,00 & 0 & 0 & 0 & 0,00 & ns \\
\hline Total & 4556 & 4380 & & 526 & & 1,88 & 384 & 350 & 384 & & \\
\hline$\chi^{2}$ & & & 51,31 & & 103,46 & & & & & & \\
\hline$P$ & & & $0,0001 * *$ & & $0,0001 * *$ & & & & & & \\
\hline
\end{tabular}


continuação

\begin{tabular}{|c|c|c|c|c|c|c|c|c|c|c|c|}
\hline \multirow{2}{*}{$\begin{array}{l}\text { DAS } \\
(\mathrm{cm})\end{array}$} & \multicolumn{3}{|c|}{ № de árvores } & \multicolumn{3}{|c|}{ Mortos } & \multirow{2}{*}{$\frac{\text { Emig. }}{\text { № }}$} & \multirow{2}{*}{$\frac{\text { Rec. }}{\text { № }}$} & \multirow{2}{*}{$\frac{\text { Imig. }}{\text { № }}$} & \multicolumn{2}{|c|}{ Cont. Poisson } \\
\hline & 1998 & 2005 & esp. & № & esp. & $\% \mathrm{ano}^{-1}$ & & & & $\mathrm{Z}$ & $P$ \\
\hline \multicolumn{12}{|c|}{ Bloco AL } \\
\hline $5<10$ & 629 & 514 & 582 & 99 & 72 & 2,61 & 60 & 38 & 6 & 8,07 & 0,001 \\
\hline $10<20$ & 279 & 308 & 258 & 10 & 32 & 0,56 & 23 & 0 & 62 & 2,98 & 0,005 \\
\hline $20<40$ & 50 & 64 & 46 & 0 & 6 & 0,00 & 3 & 0 & 17 & 3,13 & 0,002 \\
\hline $40<80$ & 1 & 2 & 1 & 0 & 0 & 0,00 & 0 & 0 & 1 & 1,00 & ns \\
\hline Total & 959 & 888 & & 109 & & 1,85 & 86 & 38 & 86 & & \\
\hline$\chi^{2}$ & & & 25,60 & & 31,25 & & & & & & \\
\hline$P$ & & & $0,0001 * *$ & & $0,0001 * *$ & & & & & & \\
\hline \multicolumn{12}{|c|}{ Bloco MG } \\
\hline $5<10$ & 439 & 383 & 407 & 66 & 51 & 2,49 & 33 & 40 & 3 & 4,70 & 0,001 \\
\hline $10<20$ & 336 & 308 & 312 & 31 & 39 & 1,48 & 31 & 0 & 34 & 2,86 & 0,005 \\
\hline $20<40$ & 111 & 130 & 103 & 7 & 13 & 1,00 & 2 & 0 & 28 & 3,12 & 0,002 \\
\hline $40<80$ & 15 & 15 & 14 & 1 & 2 & 1,06 & 0 & 0 & 1 & 0,00 & ns \\
\hline$>80$ & 1 & 1 & 1 & 0 & 0 & 0,00 & 0 & 0 & 0 & 0,00 & ns \\
\hline Total & 902 & 837 & & 105 & & 1,89 & 66 & 40 & 66 & & \\
\hline$\chi^{2}$ & & & 8,67 & & 9,17 & & & & & & \\
\hline$P$ & & & 0,0699 & & 0,0569 & & & & & & \\
\hline \multicolumn{12}{|l|}{ Bloco EJ } \\
\hline $5<10$ & 660 & 605 & 643 & 105 & 77 & 2,64 & 50 & 99 & 1 & 3,44 & 0,001 \\
\hline $10<20$ & 378 & 379 & 368 & 20 & 44 & 0,84 & 30 & 0 & 51 & 0,10 & ns \\
\hline $20<40$ & 53 & 78 & 52 & 2 & 6 & 0,59 & 2 & 0 & 29 & 4,35 & 0,001 \\
\hline $40<80$ & 2 & 3 & 2 & 0 & 0 & 0,00 & 0 & 0 & 1 & 1,00 & ns \\
\hline Total & 1093 & 1065 & & 127 & & 1,89 & 82 & 99 & 82 & & \\
\hline$\chi^{2}$ & & & 16,59 & & 26,52 & & & & & & \\
\hline$P$ & & & $0,0009 * *$ & & $0,0001 * *$ & & & & & & \\
\hline \multicolumn{12}{|c|}{ Bloco RJ } \\
\hline $5<10$ & 491 & 468 & 492 & 63 & 44 & 2,10 & 43 & 81 & 2 & 1,67 & ns \\
\hline $10<20$ & 308 & 311 & 308 & 15 & 27 & 0,77 & 25 & 0 & 43 & 0,33 & ns \\
\hline $20<40$ & 92 & 112 & 92 & 1 & 8 & 0,17 & 3 & 0 & 24 & 3,78 & 0,001 \\
\hline $40<80$ & 9 & 10 & 9 & 1 & 1 & 1,80 & 0 & 0 & 2 & 0,58 & ns \\
\hline Total & 900 & 901 & & 80 & & 1,43 & 71 & 81 & 71 & 0,06 & \\
\hline$\chi^{2}$ & & & 5,56 & & 20,53 & & & & & & \\
\hline$P$ & & & 0,1352 & & $0,0001 * *$ & & & & & & \\
\hline \multicolumn{12}{|c|}{ Bloco DR } \\
\hline $5<10$ & 432 & 390 & 424 & 87 & 65 & 3,42 & 52 & 92 & 5 & 2,73 & 0,01 \\
\hline $10<20$ & 209 & 222 & 205 & 16 & 31 & 1,22 & 24 & 0 & 53 & 1,35 & ns \\
\hline $20<40$ & 57 & 70 & 56 & 2 & 9 & 0,55 & 3 & 0 & 18 & 2,71 & 0,01 \\
\hline $40<80$ & 4 & 7 & 4 & 0 & 1 & 0,00 & 0 & 0 & 3 & 1,73 & ns \\
\hline Total & 702 & 689 & & 105 & & 2,47 & 79 & 92 & 79 & 0,69 & \\
\hline$\chi^{2}$ & & & 10,05 & & 20,80 & & & & & & \\
\hline$P$ & & & $0,0181 *$ & & $0,0001 * *$ & & & & & & \\
\hline \multicolumn{12}{|c|}{ Setor Borda } \\
\hline $5<10$ & 604 & 496 & 561 & 125 & 100 & 3,52 & 68 & 84 & 1 & 6,48 & 0,001 \\
\hline $10<20$ & 247 & 274 & 229 & 21 & 41 & 1,36 & 20 & 0 & 68 & 2,59 & 0,01 \\
\hline $20<40$ & 43 & 60 & 40 & 2 & 7 & 0,73 & 0 & 0 & 19 & 3,71 & 0,001 \\
\hline $40<80$ & 3 & 3 & 3 & 0 & 0 & 0,00 & 0 & 0 & 0 & 0,00 & ns \\
\hline Total & 897 & 833 & & 148 & & 2,75 & 88 & 84 & 88 & 3,17 & \\
\hline$\chi^{2}$ & & & 26,29 & & 20,17 & & & & & & \\
\hline$P$ & & & $0,0001 * *$ & & $0,0002 * *$ & & & & & & \\
\hline
\end{tabular}


continuação

\begin{tabular}{|c|c|c|c|c|c|c|c|c|c|c|c|}
\hline \multirow{2}{*}{$\begin{array}{l}\text { DAS } \\
(\mathrm{cm})\end{array}$} & \multicolumn{3}{|c|}{ № de árvores } & \multicolumn{3}{|c|}{ Mortos } & \multirow{2}{*}{$\frac{\text { Emig. }}{\text { № }}$} & \multirow{2}{*}{$\frac{\text { Rec. }}{\text { № }}$} & \multirow{2}{*}{$\frac{\text { Imig. }}{\text { № }}$} & \multicolumn{2}{|c|}{ Cont. Poisson } \\
\hline & 1998 & 2005 & esp. & № & esp. & $\% \mathrm{ano}^{-1}$ & & & & $\mathrm{Z}$ & $P$ \\
\hline \multicolumn{12}{|c|}{ Setor Interior } \\
\hline $5<10$ & 1547 & 1387 & 1486 & 234 & 165 & 2,50 & 125 & 188 & 11 & 6,77 & 0,001 \\
\hline $10<20$ & 990 & 976 & 951 & 57 & 106 & 0,91 & 88 & 0 & 131 & 0,84 & ns \\
\hline $20<40$ & 241 & 300 & 231 & 7 & 26 & 0,45 & 10 & 0 & 76 & 6,12 & 0,001 \\
\hline $40<80$ & 19 & 23 & 18 & 1 & 2 & 0,83 & 0 & 0 & 5 & 1,63 & ns \\
\hline$>80$ & 1 & 1 & 1 & 0 & 0 & 0,00 & 0 & 0 & 0 & 0,00 & ns \\
\hline Total & 2798 & 2687 & & 299 & & 1,73 & 223 & 188 & 223 & 3,63 & \\
\hline$\chi^{2}$ & & & 28,77 & & 65,33 & & & & & & \\
\hline$P$ & & & $0,0001 * *$ & & $0,0001 * *$ & & & & & & \\
\hline \multicolumn{12}{|c|}{ Setor Rio } \\
\hline $5<10$ & 500 & 477 & 499 & 61 & 46 & 1,99 & 45 & 78 & 5 & 1,67 & ns \\
\hline $10<20$ & 273 & 278 & 273 & 14 & 25 & 0,81 & 25 & 0 & 44 & 0,55 & ns \\
\hline $20<40$ & 79 & 94 & 79 & 3 & 7 & 0,60 & 3 & 0 & 21 & 2,89 & 0,005 \\
\hline $40<80$ & 9 & 11 & 9 & 1 & 1 & 1,80 & 0 & 0 & 3 & 1,00 & ns \\
\hline Total & 861 & 860 & & 79 & & 1,48 & 73 & 78 & 73 & 0,06 & \\
\hline$\chi^{2}$ & & & 4,45 & & 12,39 & & & & & & \\
\hline$P$ & & & 0,2171 & & $0,0062 * *$ & & & & & & \\
\hline
\end{tabular}

\section{Discussão}

O padrão apresentado em termos de dinâmica estrutural do componente arbustivo-arbóreo da floresta de galeria aluvial do Rio das Antas no intervalo de 6,5 anos foi caracterizado por taxas muito inferiores a outras formações florestais tropicais ripárias estudadas por Felfili (1995a, b), van den Berg (2001), DamascenoJunior et al. (2004), Guilherme et al. (2004), Appolinário et al. (2005) e Pinto \& Hay (2005). Provavelmente, este padrão é resultado das cheias sazonais, que inundam temporariamente as áreas de floresta e saturam hidricamente o solo aluvial limitando o estabelecimento apenas àquelas espécies capazes de suportar tal condição (Lobo \& Joly 2000, Rodrigues \& Shepherd 2000, Bertani et al. 2001, Barddal et al. 2004), sendo que freqüentemente algumas se tornam dominantes (Barddal et al. 2004). O alagamento elimina o ar do solo limitando as trocas gasosas das plantas, uma vez que poucas horas após o encharcamento todo o oxigênio contido na água já foi consumido pelas raízes e microorganismos, gerando um ambiente anóxico ou hipóxico (Lobo \& Joly 2000).

Nesta condição de saturação hídrica, as árvores, normalmente, apresentam taxa de crescimento inferior àquelas que habitam áreas mais bem drenadas (Barddal et al. 2004, Appolinário et al. 2005); os sedimentos depositados sobre os galhos e folhas pelas cheias trazem limitações adicionais ao crescimento das plantas, obstruindo a captura de luz e as trocas gasosas (Appolinário et al. 2005), além deste efeito ser mais pronunciado entre os indivíduos menores por terem maiores porções da planta submersas durante as cheias (Damasceno-Junior et al. 2004, Appolinário et al. 2005). Sendo assim, os indivíduos menores, que em condições normais já estão estressados pela deficiência em luz no sub-bosque, são mais sujeitos à fonte adicional de estresse gerada pela inundação, o que pode aumentar sua taxa de mortalidade (Appolinário et al. 2005). Talvez, estes fatores tenham atuado fortemente na determinação da maior concentração da mortalidade entre os indivíduos de menor porte $(5 \leq$ DAS $<10 \mathrm{~cm}$ ).

No período 1998-2005, a dinâmica da floresta do Rio das Antas revelou mudanças que parecem estar abrangendo a comunidade como um todo, sendo que ocorreu predomínio da mortalidade em detrimento ao recrutamento de novos indivíduos, e um acúmulo líquido em área basal. Outros autores investigando padrões de dinâmica em florestas ripárias detectaram taxas de mortalidade superiores às do presente estudo, em florestas não inundáveis (Felfili 1995a, b, van den Berg 2001), em condições de cheias normais (Guilherme et al. 2004) e muito superiores em casos de inundações excepcionais (Damasceno-Junior et al. 2004, Appolinário et al. 2005). Em florestas tropicais maduras e não perturbadas, seria esperado em longo prazo o equilíbrio entre as taxas de dinâmica, tanto em número de indivíduos 
como em área basal, sendo que este equilíbrio indicaria uma estabilidade estrutural para a comunidade arbórea (Lieberman et al. 1985, Swaine et al. 1987, Rankin-deMerona et al. 1990, Felfili 1995a, Santos et al. 1998).

Entre as causas de períodos de instabilidade, alguns autores destacam as flutuações cíclicas, que atuam causando desequilíbrio em florestas maduras, e após sucessivos períodos em que mortalidade ou recrutamento, e perda ou ganho em biomassa, aumentam ou diminuem, retornam a uma condição de equilíbrio (Swaine 1990, Felfili 1995a). Appolinário et al. (2005) destacaram no Rio Grande, no sul de Minas Gerais, um possível exemplo de ciclo rítmico ocasionado por cheias anormais, as quais têm recorrência em um intervalo médio de 15 a 35 anos, sendo que esse impacto natural pode condicionar fortes variações temporais na dinâmica florestal. Cabe ressaltar que em condições de eventos de cheias regulares, teoricamente, os efeitos seriam absorvidos pela comunidade (Damasceno-Junior et al. 2004). Neste aspecto, DamascenoJunior et al. (2004) verificaram no Rio Paraguai, Corumbá (MT), que após uma cheia excepcional a mortalidade de indivíduos foi muito superior nos sítios que não são normalmente atingidos por cheias, enquanto nos locais onde as inundações ocorrem com maior freqüência esse impacto foi menos intenso. Felfili (1995a) ressaltou que variações climáticas podem causar períodos de instabilidade para a comunidade florestal, porém nesse caso os sistemas florestais seriam afetados em uma área muito mais extensa.

Além dos fatores supracitados, outros podem atuar como fontes de distúrbios na dinâmica de sistemas florestais, destacando-se os incêndios, deslizamentos de terra, tempestades, formação de clareiras, secas, inundações e, também impactos de origem antrópica, entre eles o desmatamento e o pastoreio pelo gado (Crow 1980, van den Berg 2001, Damasceno-Júnior et al. 2004, Guilherme et al. 2004). Na floresta do Rio das Antas, as inundações constituem impactos que atingem ciclicamente a floresta como um todo, porém nos blocos estudados, principalmente no $\mathrm{AL}$, foram detectadas diversas cepas provenientes de cortes seletivos de madeira anteriores a 1998. Esse impacto teria gerado alterações na estrutura da floresta e atuado como uma, senão a principal, causa do estágio atual em que se encontra a dinâmica da floresta neste bloco. Supostamente, essa retirada de madeira teria resultado, anteriormente a 1998-2005, em uma fase de "construção inicial", devido ao aumento repentino da disponibilidade de luz nos pontos da floresta onde as árvores foram suprimidas, gerando aumento simultâneo da densidade e da área basal. Posteriormente, entre 1998 e 2005, a floresta estaria atravessando uma etapa de "construção tardia", devido ao processo de autodesbaste no componente arbustivo-arbóreo, com aumento da mortalidade de indivíduos (principalmente na menor classe diamétrica) e redução do recrutamento e engrossamento (acúmulo de biomassa) dos sobreviventes (Chagas et al. 2001, Appolinário et al. 2005). As regressões testadas evidenciam o processo de autodesbaste descrito anteriormente, visto que nas parcelas mais densas em 1998 ocorreu declínio da densidade com perda em área basal mais acentuada (devido à morte de indivíduos). Em um estágio seguinte, essa área da floresta tenderia a uma maior maturidade. Porém, cabe uma ressalva quanto à sucessão da floresta do Rio das Antas para um estágio de estabilidade, especialmente no trecho do bloco MG, onde o tráfego intenso do gado é provavelmente a principal causa da redução significativa no número de indivíduos com tamanho inferior a $20 \mathrm{~cm}$ de DAS. A presença do gado neste trecho da floresta provavelmente afetou a sobrevivência dos indivíduos menores devido ao pisoteio, além de ter aumentado a compactação do solo, o que dificultou o estabelecimento e desenvolvimento radicular dos indivíduos jovens. A continuidade sistemática desse impacto, o qual persiste até os dias atuais, poderia resultar futuramente em uma lacuna de indivíduos aptos a habitar o dossel superior da floresta, resultando em médio e longo prazo num empobrecimento da floresta no trecho compreendido por esse bloco.

O padrão de dinâmica da floresta de galeria aluvial do Rio das Antas apresentou uma diferenciação em relação ao distanciamento do rio e proximidade da borda. A maior rotatividade do estrato arbustivo-arbóreo da floresta no setor Borda, com redução líquida de indivíduos e acúmulo de área basal, foi incrementada pela provável composição predominante de espécies de ciclo de vida curto e que demandam por luz (Felfili 1995a, b, Kellman et al. 1998, van den Berg 2001, Oliveira-Fiho et al. 2007), sendo que a intensidade luminosa, apesar de não ter sido mensurada, aparentemente diferiu entre os setores. Corroborando com esta expectativa, van den Berg \& Santos (2003) avaliando uma floresta de galeria em Itutinga, MG, verificaram que a abertura do dossel reduz com o distanciamento da borda da floresta em direção ao interior, resultando em diferenças na intensidade luminosa entre os setores. Em bordas recentes seria esperado um aumento repentino na densidade nos primeiros anos pós-distúrbio, devido ao aumento das taxas de incidência luminosa e temperatura, maior penetração dos ventos, menor umidade relativa do ar e do solo (van den Berg \& Santos 2003). Portanto, pode-se considerar esta pequena redução na densidade, ou seja, a relativa estabilidade, como um indicador de que esta borda seja 
antiga, fato comprovado também através da comparação entre ortofotos de 1952 e 1997, as quais mostram que a floresta do Rio das Antas não sofreu mudanças significativas em seus limites (E. van den Berg dados não publicados).

Essa heterogeneidade espacial da dinâmica pode ser resultado também de fatores topográficos, pois se nota a presença de uma micro-elevação no setor marginal ao curso d'água, formando um dique sedimentar. Nas áreas de dique, a inundação tem duração de poucas horas, no máximo alguns dias, com uma renovação contínua de água (Lobo \& Joly 2000), resultando em menor impacto na aeração do solo, e permitindo o estabelecimento de um grupo mais amplo de espécies. Assim, é provável que o micro-relevo encontrado na floresta do Rio das Antas resulte em uma mais intensa saturação hídrica do solo no setor Borda e em grande parte das parcelas do setor Interior, que estão topograficamente abaixo do dique. Comprovando essa maior saturação, em determinados locais, adjacentes à Borda da floresta ocorre a formação de lagoas temporárias ou de charcos. Algumas destas lagoas podem permanecer cheias por longos períodos, sendo que a água fica estagnada, com movimentação somente no sentido vertical, seja pela elevação do nível do lençol freático e, ou, pelo represamento de água extravasada do rio (Lobo \& Joly 2000).

Os padrões de diferenciação espacial da dinâmica da comunidade da floresta de galeria aluvial do Rio das Antas refletem, provavelmente, a variação da disponibilidade de luz entre os setores da floresta, e também a variação da saturação hídrica do solo. Em função da saturação hídrica do solo e da topografia "plana" (a elevação do lençol freático, nos períodos de cheia, impacta a floresta como um todo) era esperado que essa floresta apresentasse taxas de dinâmica inferiores a outras formações florestais ripárias, o que foi evidenciado pelo presente estudo. Porém novos estudos devem ser conduzidos em florestas aluviais, além da continuidade que deve ser dada ao presente estudo, a fim de confirmar o padrão aqui encontrado. Os impactos causados aos indivíduos das duas classes diamétricas menores pelo tráfego do gado no bloco MG revelam que cuidados adicionais devem ser adotados visando impedir o acesso desses animais ao interior de floresta, sob pena de, em médio ou longo prazo, a regeneração estrutural da floresta ser afetada significativamente, resultando em empobrecimento e "raleamento" do estrato arbustivoarbóreo. É importante que a pesquisa seja também direcionada à dinâmica populacional, principalmente, com objetivo de subsidiar informações necessárias para a recuperação de florestas neste ambiente.
Agradecimentos - Ao Departamento de Saúde, Segurança e Meio Ambiente da Alcoa Alumínio S.A., unidade de Poços de Caldas, MG pelo apoio financeiro concedido.

\section{Referências bibliográficas}

APPOLINÁRIO, V., OLIVEIRA-FILHO, A.T. \& GUILHERME, F.A.G. 2005. Tree population dynamics in a Brazilian tropical semideciduous forest. Revista Brasileira de Botânica 28:347-360.

BARDDAL, M.L., RODERJAN, C.V., GALVÃO, F. \& CURCIO, G.R. 2004. Caracterização florística e fitossociológica de um trecho sazonalmente inundável de floresta aluvial, em Araucária, PR. Santa Maria, Ciência Florestal 14:37-50.

BERTANI, D.F., RODRIGUES, R.R., BATISTA, J.L.F. \& SHEPHERD, G.J. 2001. Análise temporal da heterogeneidade florística e estrutural em uma floresta ribeirinha. Revista Brasileira de Botânica 24:11-23.

CHAGAS, R.K., OLIVEIRA-FILHO, A.T., VAN DEN BERG, E. \& SCOLFORO, J.R.S. 2001 Dinâmica de populações arbóreas em um fragmento de floresta estacional semidecidual montana em Lavras, Minas Gerais. Revista Árvore 25:39-57.

CROW, T.R. 1980. A rainforest chronicle: a 30-year record of change in structure and composition at El Verde, Puerto Rico. Biotropica 12:42-55.

DAMASCENO-JUNIOR, G.A., SEMIR, J., SANTOS, F.A. M. \& LEITÃO-FILHO, H.F. 2004. Tree mortality in a riparian forest at rio Paraguai, Pantanal, Brazil, after an extreme flooding. Acta Botanica Brasilica 18:839-846.

EMBRAPA 2000. Sistema brasileiro de classificação de solos. $2^{a}$ ed. Rio de Janeiro.

FELFILI, J.M. 1995a. Growth, recruitment and mortality in the Gama gallery forest in central Brazil over a six-year period (1985-1991). Journal of Tropical Ecology 11:67-83.

FELFILI, J.M. 1995b. Diversity, structure and dynamics of a gallery forest in central Brazil. Vegetatio 117:1-15

GUILHERME, F.A.G., OLIVEIRA-FILHO, A.T., APPOLINÁRIO, V. \& BEARZOTI, E. 2004. Effects of flooding regimes and woody bamboos on tree community dynamics in a section of tropical semideciduous forest in South-Eastern Brazil. Plant Ecology 174:19-36.

JACOMINE, P.K.T. 2000. Solos sob matas ciliares. In Matas ciliares: Conservação e recuperação (R.R. Rodrigues \& H.F. Leitão-Filho, eds.). Editora da Universidade de São Paulo, São Paulo, p.27-31.

KELLMAN, M., TACKABERRY, R. \& RIGG, L. 1998. Structure and function in two tropical gallery forest communities: implications for forest conservation in fragmented systems. Journal of Applied Ecology 35: 195-206.

KORNING, J. \& BALSLEV, H. 1994. Growth and mortality of trees in Amazonian tropical rain forest in Ecuador. Journal of Vegetation Science 4:77-86. 
LAURANCE, W.F., FERREIRA, L.V., RANKIN-DEMERONA, J.M. \& LAURANCE, S.G. 1998. Rain forest fragmentation and the dynamics of Amazonian tree communities. Ecology 79:2032-2040.

LIEBERMAN, D., LIEBERMAN, M., PERALTA, R. \& HARTSHORN, G.S. 1985. Mortality patterns and stand turnover rates in a wet tropical forest in Costa Rica. Journal of Ecology 73:915-924.

LOBO, P.C. \& JOLY, C.A. 2000. Aspectos ecofisiológicos da vegetação de mata ciliar do Sudeste do Brasil. In Matas ciliares: Conservação e recuperação (R.R. Rodrigues \& H.F. Leitão-Filho, eds.). Editora da Universidade de São Paulo, São Paulo, p.143-157.

NASCIMENTO, H.E.M., DIAS, A.S., TABANEZ, A.A.J. \& VIANA, V.M. 1999. Estrutura e dinâmica de populações arbóreas de um fragmento de floresta estacional semidecidual na região de Piracicaba, SP. Revista Brasileira de Biologia 59:329-342.

OLIVEIRA-FILHO, A.T., MELLO, J.M. \& SCOLFORO, J.R.S. 1997. Effects of past disturbance and edges on tree community structure and dynamics within a fragment of tropical semideciduous forest in southeastern Brazil over a five year period (1987-1992). Plant Ecology 131:45-66.

OLIVEIRA-FILHO, A.T., CARVALHO, W.A.C., MACHADO, E.L.M., HIGUCHI, P., CASTRO, G.C., SILVA, A.C., SANTOS, R.M., BORGES, L.F.R., CORREAA, B.S. \& ALVES, J.M. 2007. Dinâmica da comunidade e populações arbóreas da borda e interior de um remanescente florestal na Serra da Mantiqueira, Minas Gerais, em um intervalo de cinco anos (19992004). Revista Brasileira de Botânica 30:149-161.

PAGANO, S.N., LEITÃO-FILHO, H.F. \& CAVASSAN, O. 1995. Variação temporal da composição florística e estrutura fitossociológica de uma floresta mesófila semidecídua, Rio Claro, Estado de São Paulo. Revista Brasileira de Biologia 55:241-258.

PINTO, J.R.R. \& HAY, J.D.V. 2005. Mudanças florísticas e estruturais na comunidade arbórea de uma floresta de vale no Parque Nacional da Chapada dos Guimarães, Mato Grosso, Brasil. Revista Brasileira de Botânica 28:523-539.

PREFEITURA MUNICIPAL DE POÇOS DE CALDAS 1992. Plano diretor do município de Poços de Caldas. http://www. pocosdecaldas.mg.gov.br (acesso em 03/ 07/2007).

RADAMBRASIL 1983. Levantamento de recursos naturais. Ministério das Minas e Energia, Rio de Janeiro.
RANKIN-DE-MERONA, J.M., HUTCHINGS, H.R. \& LOVEJOY, T.E. 1990. Tree mortality and recruitment over a five-year period in undisturbed upland rainforest of the Central Amazon. In Four Neotropical rainforests (A.H. Gentry, ed.). Yale University Press, New Haven, p.573-584.

RIBEIRO, J.F. \& WALTER, B.M.T. 1998. Fitofisionomias do bioma Cerrado. In Cerrado: Ambiente e flora (S.M. Sano \& S.P. Almeida, eds.). Embrapa-Cipac, Planaltina, p.89-166.

RODRIGUES, R.R. \& SHEPHERD, G.J. 2000. Fatores condicionantes da vegetação ciliar. In Matas ciliares: Conservação e recuperação (R.R. Rodrigues \& H.F. Leitão-Filho, eds.). Editora da Universidade de São Paulo, São Paulo, p.101-107.

SANTOS, F.A.M., PEDRONI, F., ALVES, L.F. \& SANCHEZ, M. 1998. Structure and dynamics of tree species of the Atlantic Forest. Anais da Academia Brasileira de Ciências 70:874-880.

SHEIL, D., BURSLEM, D.F.R.P. \& ALDER, D. 1995. The interpretation and misinterpretation of mortality rate measures. Journal of Ecology 83:331-333.

SHEIL, D., JENNINGS, S. \& SAVILL, P. 2000. Long-term permanent plot observations of vegetation dynamics in Budongo, a Ugandan rain forest. Journal of Tropical Ecology 16:765-800.

SOKAL, R.R. \& ROHLF, F.J. 1995. Biometry. 3a ed. Freeman \& Co, Nova York.

SWAINE, M.D., LIEBERMAN, D. \& PUTZ, F.E. 1987. The dynamics of tree populations in tropical forest: a review. Journal of Tropical Ecology 3:359-366.

SWAINE, M.D. 1990. Population dynamics of tree species in tropical forests. In Tropical forests: botanical dynamics, speciation and diversity (L.B. Holm-Nielsen, I.C. Nielsen \& H. Balslev, eds.). Academic Press, London, p.101-110.

VAN DEN BERG, E. 2001. Variáveis ambientais e a dinâmica estrutural e populacional de uma floresta de galeria em Itutinga, MG. Tese de doutorado, Universidade Estadual de Campinas, Campinas.

VAN DEN BERG, E. \& SANTOS, F.A.M. 2003. Aspectos da variação ambiental em uma floresta de galeria em Itutinga, MG, Brasil. Ciência Florestal 13:83-98.

WERNECK, M. \& FRANCESCHINELLI, E.V. 2004. Dynamics of a dry forest fragment after the exclusion of human disturbance in southeastern Brazil. Plant Ecology 174:337-346.

ZAR, J.H. 1996. Biostatistical analysis. 3a ed. Prentice-Hall, New Jersey. 